\title{
CONTRIBUCIÓN AL CONOCIMIENTO DE LOS MURCIÉLAGOS (MAMMALIA, CHIROPTERA) DEL PARQUE NATURAL DE PENYAGOLOSA (PROVINCIA DE CASTELLÓN)
}

\author{
Joaquín Albesa ${ }^{1, *} \&$ Jacques Ros $^{2}$ \\ 1,*Departamento de Geología. Universitat de València. C/ Dr. Moliner, 50. E-46100 Burjassot. España. \\ E-mail: joaquin.albesa@uv.es - ORCID-iD: https://orcid.org/0000-0002-7318-8396 \\ ${ }^{2} 2$ impasse Psalette. F-56000 Vannes. Francia. E-mail: rosjacques5@gmail.com - \\ ORCID-iD: https://orcid.org/0000-0003-3150-3842 \\ * Autor para correspondencia.
}

\section{RESUMEN}

Se exponen los principales resultados obtenidos en los muestreos efectuados en agosto de 2004 en el Parque Natural de Penyagolosa. La comunidad de quirópteros del Parque Natural está integrada por al menos 14 especies. Diez de ellas fueron localizadas en los muestreos, siendo ocho nuevas citas tras ellos. La revisión de la bibliografía e informes técnicos más recientes permiten añadir las restantes cuatro especies. De entre las especies localizadas en los muestreos, destacan las forestales Plecotus auritus (Linnaeus, 1758) y Barbastella barbastellus (Schreber, 1774); las fisurícolas Pipistrellus pipistrellus (Schreber, 1774) y Eptesicus serotinus (Schreber, 1774) y la cavernícola Myotis escalerai Cabrera, 1904. Plecotus auritus y Pipistrellus pipistrellus son las especies mayormente localizadas en consonancia, en la primera de ellas, con el carácter netamente forestal del Parque Natural y, en la segunda, con su carácter generalista y por ello ser la especie más abundante en el interior de la Comunidad Valenciana. La información obtenida sobre P. auritus y B. barbastellus confirma la presencia de poblaciones en un contexto de elevado valor biogeográfico al tratarse, en la primera de las especies, de una población situada en el límite meridional de su área de distribución y, en la segunda de ellas, al ser una especie con una distribución restringida a determinados ambientes forestales del interior.

Palabras clave: Chiroptera; Penyagolosa; Castellón; España; Distribución.

\section{ABSTRACT}

Contribution to the knowledge of the bats (Mammalia, Chiroptera) of the Natural Park of Penyagolosa (province of Castellón)

The main results obtained in the samplings carried out in August 2004 in the Penyagolosa Natural Park are presented. The community of bats of the Natural Park is made up of at least 14 species. Ten of them were located in the samplings, being eight new findings. The review of the most recent bibliography and technical reports allow the remaining four species to be added. Among the species located in the samplings, stand out the forest ones Plecotus auritus (Linnaeus, 1758) and Barbastella barbastellus (Schreber, 1774) stand; the crevice-dwelling Pipistrellus pipistrellus (Schreber, 1774) and Eptesicus serotinus (Schreber, 1774), and the cave-dwelling Myotis escalerai Cabrera, 1904. Plecotus auritus and Pipistrellus pipistrellus are the most commonly located species. The first one due to purely forest character of the Natural Park and, the second, because of its generic nature-it is therefore, the most abundant species in the interior of the Valencian Community. The information obtained on $P$. auritus and $B$. barbastellus confirms the presence of their populations in a context of high biogeographical value since first of the species, is a population located in the southern limit of their distribution area, and, the second is a species with a restricted distribution to certain forest environments of the interior.

Keywords: Chiroptera; Penyagolosa; Castellón; Spain; Distribution.

Cómo citar este artículo/Citation: Albesa, J. \& Ros, J. 2019. Contribución al conocimiento de los murciélagos (Mammalia, Chiroptera) del Parque Natural de Penyagolosa (provincia de Castellón). Grael/sia, 75(1): e090. https://doi.org/10.3989/ graellsia.2019.v75.198 


\section{Introducción}

El macizo de Penyagolosa se sitúa entre el oeste de la provincia de Castellón y el este de la provincia de Teruel. Posee una superficie de más de $150 \mathrm{~km}^{2}$ repartidos entre los municipios castellonenses de Vistabella del Maestrat, Xodos, Llucena del Cid, Castillo de Villamalefa y Villahermosa del Río y el municipio turolense de Puertomingalvo. El macizo cuenta con la máxima elevación de la provincia de Castellón en el pico de Penyagolosa (1.814 m.s.n.m.), así como con numerosas cimas que superan los 1.500 m.s.n.m. En el seno del macizo (Fig. 1), se encuentra localizado el Parque Natural de Penyagolosa. La localización geográfica del área declarada como Parque Natural, su altitud y climatología asociada, así como sus particularidades geomorfológicas y edafológicas posibilitan la existencia comunidades biológicas que cuentan con importantes elementos relictos como es el caso de la monocotiledónea Galanthus nivalis L. (Fabregat Llueca et al., 2005), el lepidóptero Graellsia isabellae (Graells, 1849) (Font Bustos, 1978) y el lacertido Podarcis muralis (Laurenti, 1768) (Pérez y de Lanuza et al., 2012).

Los murciélagos constituyen, con 31 especies (Palomo et al., 2007), el orden más numeroso dentro de los vertebrados de la Península Ibérica. Para la zona que nos ocupa, los estudios existentes corresponden a trabajos desarrollados en el seno de diversos proyectos entre los que destaca un proyecto LIFE (LIFE00 / NAT/7337) y otros como los de Almenar \& Alcocer (1998), Alcocer et al. (2001) y Albesa \& Ros (2005). Sin embargo, la información publicada se reduce a dos trabajos específicos (Almenar \& Alcocer, 2000; Alcocer et al., 2003) y a algunos más amplios entre los que destacan Monsalve et al. (2003) y la parte dedicada a los quirópteros en una monografía sobre los mamíferos de la Comunidad Valenciana (en adelante CV) (Jiménez et al., 2012).
El presente trabajo pretende contribuir a mejorar el conocimiento de los murciélagos, especialmente los forestales, del Parque Natural de Penyagolosa, mediante la publicación de los resultados obtenidos en el seno de un proyecto sobre los murciélagos de los macizos montañosos de la provincia de Castellón (Albesa \& Ros, 2005), a la vez que aportar un punto de referencia para futuros estudios que busquen valorar el estado y evolución de las poblaciones.

\section{Material y métodos}

El Parque Natural de Penyagolosa fue declarado, a través del DECRETO 50/2006 del Consell de la Generalitat Valenciana, en abril de 2006 sobre una superficie de 1.094,45 ha. El Parque Natural se caracteriza por poseer una orografía abrupta sobre un territorio que, comprendido entre las cotas $1.250 \mathrm{y}$ 1.814 m.s.n.m., destaca por los numerosos barrancos, por los importantes desniveles de su parte meridional y por los relieves más suaves de la zona septentrional. La combinación de su ubicación geográfica y su altitud propician unos valores climáticos estudiados por Vigo (1968), quien calcula, entre otros valores, las medias anuales de precipitación $(747 \mathrm{~mm}$ ) y temperatura $\left(8,3^{\circ} \mathrm{C}\right)$, así como el índice de Emberger, cuyo valor (145) corresponde, según indica, a un clima mediterráneo húmedo.

La vegetación del Parque Natural está dominada en las cotas más bajas y meridionales por la asociación Quercetum rotundifoliae, cuyo principal exponente es la carrasca (Quercus rotundifolia Lam.). En las cotas medias predominan los pinares de Pinus sylvestris L. y Pinus nigra J.F. Arnold que, en las zonas más húmedas, van acompañados de Acer opalus Mill., Ilex aquifolium L., Tilia platyphyllos Scop. y Taxus baccata L., entre otros. Destacable también en esta cota es la presencia de Quercus faginea Lam. en suelos básicos y de Quercus pyrenaica Willd. en suelos ácidos. En las

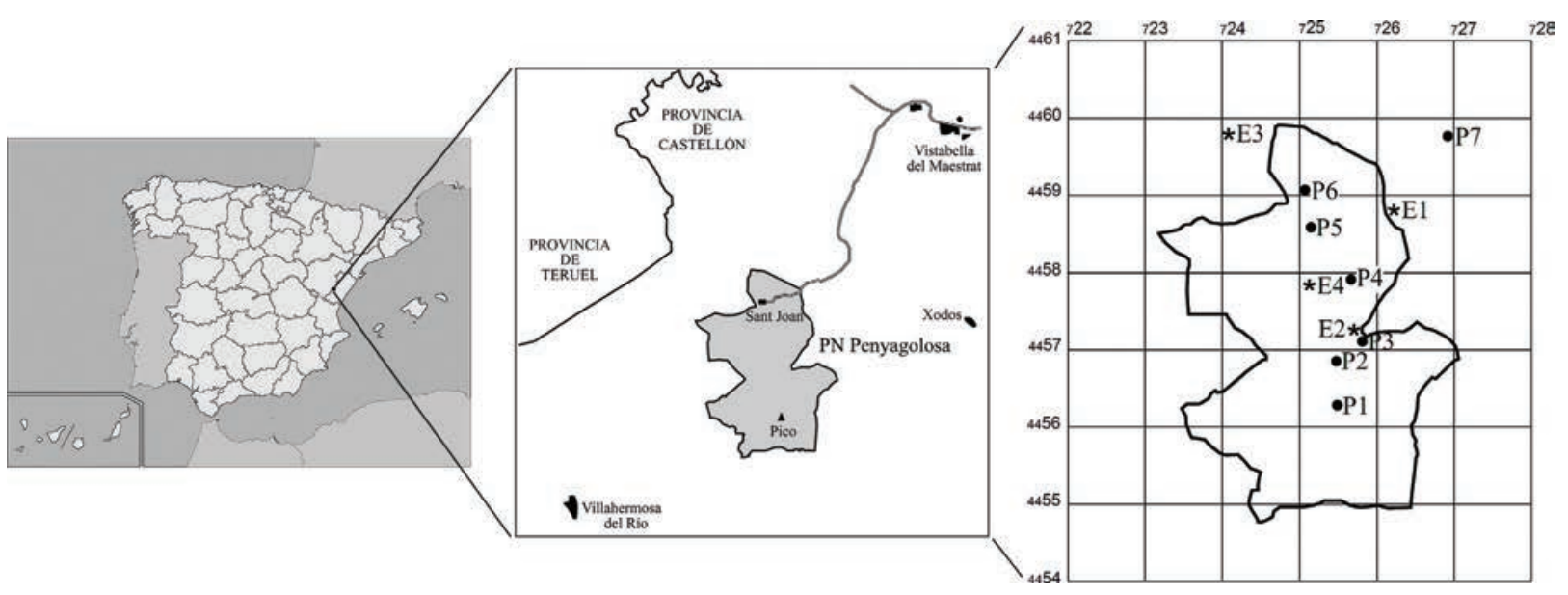

Fig. 1.- Localización del Parque Natural de Penyagolosa, las estaciones de muestreo (E) y las paradas del transecto (P).

Fig. 1.- Location of the Natural Park of Penyagolosa, the sampling stations (E) and the acoustic transect stops (P). 
cotas más elevadas, la vegetación dominante corresponde principalmente a ejemplares aislados de Pinus sylvestris, acompañados de Juniperus sabina L., y a un tapiz de Erinacea anthyllis Link.

El trabajo de campo se realizó durante el mes de agosto de 2004, utilizándose de forma complementaria capturas con redes y observaciones acústicas con un detector de ultrasonidos modelo Pettersson Elektronik D980 en un uso combinado de metodologías, lo que posibilita incrementar la detección de especies (Flaquer et al., 2007).

Los registros se obtuvieron en cuatro estaciones y a lo largo de un transecto, constando la denominación, localización y principales características de las estaciones y de las paradas en la Figura 1 y en la Tabla 1.

En las estaciones se emplearon redes de distinta longitud en función de las características de la zona, siendo las dimensiones utilizadas 3, 6, 9 y $12 \mathrm{~m}$ de longitud y $2 \mathrm{~m}$ de altura. El muestreo, que comenzó siempre alrededor de las 21:00 horas, tuvo una duración que osciló entre una y media y dos horas. En todos los ejemplares capturados, siguiendo los criterios de Schober \& Grimmberger (1996) y Dietz \& von Helversen (2004), se efectuó la determinación taxonómica hasta el nivel de especie; se observó su sexo y edad; se estimó su peso y se tomaron medidas biométricas. Todos los murciélagos fueron liberados tras la toma de datos en la misma noche y estación donde fueron capturados, si bien fueron custodiados en bolsas de tela hasta el fin del muestreo para evitar fuesen recapturados. En todas las estaciones se realizó un promedio de tres escuchas, previo al comienzo de capturas, durante ellas y una vez finalizadas, con una duración de diez minutos cada una y un total de 120 minutos acumulados para el conjunto de las estaciones.

El transecto cubría inicialmente una distancia de aproximadamente $15 \mathrm{~km}$ y contaba con 10 paradas en las que previamente se habían identificado ambientes diferentes a partir de las características de la vegetación, la orografía, la altitud y el grado de antropización. Sin embargo, tras la declaración de la figura de Parque Natural sólo siete de las paradas se encuentran localizadas dentro de los límites de la zona de protección del Parque Natural o en las cuadrículas inmediatamente adyacentes y, por ello, en este trabajo, no han sido consideradas las tres últimas. El recorrido del transecto se realizó entre las 21:23 y 23:40 horas, permaneciendo escuchando durante diez minutos en cada parada y, con ello, un total de 70 minutos en todo el conjunto del transecto.

La determinación de los contactos se efectuó observando el tipo de pulso, su frecuencia de máxima energía, su duración y su ritmo (Ahlén, 1990; Barataud, 1996, 2002; Russo \& Jones, 2002).

Ante la posibilidad de que un mismo ejemplar fuese escuchado en más de una ocasión, se adoptaron medidas para evitar reconteos. En ese sentido, apoyados en el reducido número de contactos registrados, sólo se consideraron los contactos que con seguridad correspondían a ejemplares diferentes al coincidir éstos en el tiempo. En todo caso, en consonancia con lo expuesto por Barataud (2012), los resultados de las escuchas se consideraron sólo en términos cualitativos, así como base del cálculo de los índices de actividad. En el caso de especies correspondientes a los

Tabla 1.- Estaciones de muestreo y paradas del transecto acústico.

Table 1.- Sampling stations and acoustic transect stops.

\begin{tabular}{|c|c|c|c|c|c|c|c|}
\hline Fecha & $\begin{array}{l}\text { Estación }(E)-P a r a d a \\
\text { transecto }(P)\end{array}$ & Horario & $\begin{array}{c}\text { UTM X } \\
\text { (ETRS89) }\end{array}$ & $\begin{array}{c}\text { UTM Y } \\
\text { (ETRS89) }\end{array}$ & $\begin{array}{c}\text { Altitud } \\
\text { (m.s.n.m.) }\end{array}$ & $\mathbf{T}^{\mathrm{a}}\left({ }^{\circ} \mathrm{C}\right)$ & Ambiente \\
\hline $19-8-2004$ & E1 Bco.del Avellanar & $21: 15-23: 15$ & 726231,66 & 4458859,93 & 1275 & 12.0 & $\begin{array}{l}\text { Bosque de Pinus nigra y Pinus } \\
\text { sylvestris }\end{array}$ \\
\hline 20-8-2004 & E2 Balsa de la Bañadera & $21: 15-22: 45$ & 725722,92 & 4457187,96 & 1490 & 10.0 & $\begin{array}{l}\text { Balsa abierta entre Pinus } \\
\text { sylvestris }\end{array}$ \\
\hline $22-8-2004$ & E3 El Rebollar & $21: 15-23: 15$ & 724002,41 & 4459767,19 & 1445 & 15.6 & $\begin{array}{l}\text { Espacio abierto próximo a } \\
\text { robledal de Quercus pyrenaica }\end{array}$ \\
\hline 21-8-2004 & P1 Ladera de Penyagolosa & $21: 23-21: 33$ & 725553,53 & 4456308,55 & 1573 & 12.0 & Pinus sylvestris dispersos \\
\hline $21-8-2004$ & P2 El Corralico & $21: 49-21: 59$ & 725532,50 & 4456829,78 & 1508 & 12.3 & Bosque de Pinus sylvestris \\
\hline $21-8-2004$ & P3 La Banyadera & 22:06-22:16 & 725750,25 & 4457031,79 & 1490 & 10.1 & Bosque de Pinus sylvestris \\
\hline $21-8-2004$ & P4 Bastón del gigante & $22: 36-22: 46$ & 725690,06 & 4457957,96 & 1410 & 13.6 & Bosque de Pinus sylvestris \\
\hline $21-8-2004$ & P5 Barranco de la Pegunta & $22: 55-23: 05$ & 725216,45 & 4458563,86 & 1256 & 11.5 & Bosque de Pinus nigra \\
\hline $21-8-2004$ & P6 Sant Joan & $23: 12-23: 22$ & 725034,28 & 4458986,28 & 1274 & 11.6 & $\begin{array}{l}\text { Espacio abierto con } \\
\text { construcciones }\end{array}$ \\
\hline $21-8-2004$ & P7 Carretera & $23: 30-23: 40$ & 726977,15 & 4459775,56 & 1223 & 11.2 & $\begin{array}{l}\text { Bosque de Pinus sylvestris y } \\
\text { Pinus nigra }\end{array}$ \\
\hline $22-8-2004$ & E4 Fuente de la Pegunta & 21:15-23:15 & 725224,39 & 4457906,37 & 1336 & 13.8 & $\begin{array}{l}\text { Bosque laxo de Pinus sylvestris } \\
\text { con pequeño curso de agua }\end{array}$ \\
\hline
\end{tabular}


géneros Plecotus y Myotis de pequeño tamaño caracterizadas por ser crípticas acústicamente, se decidió determinarlas como Plecotus auritus/austriacus y Myotis sp. Dado que la detectabilidad de las especies varía en función de la intensidad de la señal y la atenuación que ésta puede sufrir, sobre los contactos brutos obtenidos se calcularon los contactos ponderados al aplicar un coeficiente de detectabilidad relacionado con la distancia de percepción en entornos forestales (Barataud, 2012).

La localización de cada especie se ha representado sobre mapas con cuadrícula $1 \mathrm{~km} \times 1 \mathrm{~km}$, incluyéndose también en estos las citas bibliográficas (Fig. 2).

Con la información obtenida en los muestreos se calcularon diversos estadísticos.

Para valorar la contribución de cada método de estudio, se analizaron el número de capturas y de contactos obtenidos, así como el número de taxones que representaban cada uno de ellos.

Con el objeto de valorar la presencia de cada especie e inferir preferencias de uso del entorno, se contabilizó el número de ejemplares capturados y se calculó su porcentaje en cada estación y en el conjunto de ellas, así como el éxito de captura de cada estación a partir del cociente entre el número de ejemplares capturados y el esfuerzo realizado, entendiendo este último como el producto del tiempo que duró el muestreo por los metros lineales de red instalados. Análogamente, se contabilizó el número de contactos acústicos en cada estación para, junto con las capturas, calcular la constancia de cada especie en cada estación. Con los contactos acústicos de cada estación y los del transecto se calculó el índice de actividad correspondiente al número de contactos por hora. Dicho índice fue calculado a partir del número de contactos registrados dividido por el tiempo total de escucha previa aplicación de los coeficientes de detectabilidad sugeridos por Barataud (2012) para ambientes forestales.

\section{Resultados}

Los muestreos efectuados han aportado 50 registros de los que 46 corresponden a 10 taxones de rango específico, tres a un grupo y uno a un par de especies:

\footnotetext{
Rhinolophus ferrumequinum (Schreber, 1774)

Myotis escalerai Cabrera, 1904

Myotis sp.

Eptesicus serotinus (Schreber, 1774)

Pipistrellus pipistrellus (Schreber, 1774)

Pipistrellus pygmaeus (Leach, 1825)

Pipistrellus kuhlii (Kuhl, 1817)

Hypsugo savii (Bonaparte, 1837)

Plecotus auritus (Linnaeus, 1758)

Plecotus auritus/austriacus
}

\section{Barbastella barbastellus (Schreber, 1774) \\ Tadarida teniotis (Rafinesque, 1814)}

La metodología que aportó mayor número de registros fue la captura con redes, al suministrar información sobre 29 ejemplares correspondientes al 58,33\% de los taxones localizados en los muestreos. Sin embargo, aunque con un menor número de registros brutos, las escuchas aportaron información sobre el $75 \%$ de los taxones (Tabla 2).

Siete de las especies registradas en los muestreos del presente estudio fueron detectadas mediante la captura con redes en las estaciones, siendo la estación de la Balsa de la Banyadera la que, con 20 ejemplares de cinco especies y un ritmo de captura de un individuo cada 4,5 minutos, registró con un valor de 27,77 un mayor éxito de captura. Las detecciones acústicas en las propias estaciones registraron también siete taxones, coincidiendo cuatro de las especies con las capturadas y siendo dos nuevas más una posible tercera al considerar el registro de $P$. auritus/austriacus. El conjunto de las capturas y de los registros acústicos en las estaciones posibilitó, por tanto, detectar nueve especies más el par $P$. auritus/austriacus. El transecto acústico permitió detectar cuatro taxones, coincidiendo dos de ellos con especies registradas en las estaciones ( $P$. pistrellus y E. serotinus) y siendo dos nuevos (Myotis sp. y T. teniotis), lo que implica la adición de una especie y un taxón genérico al conjunto de los muestreos, situándolo en al menos diez especies. Los registros concretos e índices calculados se muestran en la Tabla 2.

Se expone a continuación la información más relevante correspondiente a las especies localizadas agrupadas por el refugio que habitualmente utilizan.

\section{ESPECIES FORESTALES}

Por lo que se refiere a las especies forestales (P. auritus y $B$. barbastellus), se trata del grupo mayormente localizado en los muestreos. Las 20 capturas que corresponden a las dos especies han supuesto el $68,97 \%$ de las obtenidas.

Plecotus auritus fue la especie más abundante en las estaciones con una constancia del $75 \%$ y el $58,62 \%$ de las capturas. El registro acústico obtenido en una de las estaciones y atribuido al par $P$. auritus/ austriacus podría corresponder también a $P$. auritus. Sin embargo, la simpatría que muestran ambas especies (Ashrafi et al., 2013) aconseja no asignar el registro a la primera a la vez que observar la posibilidad de que correspondiese a la segunda.

Barbastella barbastellus fue localizada en dos de las cuatro estaciones de muestreo a través de tres capturas que constituyen el 10,35\% de las efectuadas, así como mediante un registro acústico en una de ellas. 

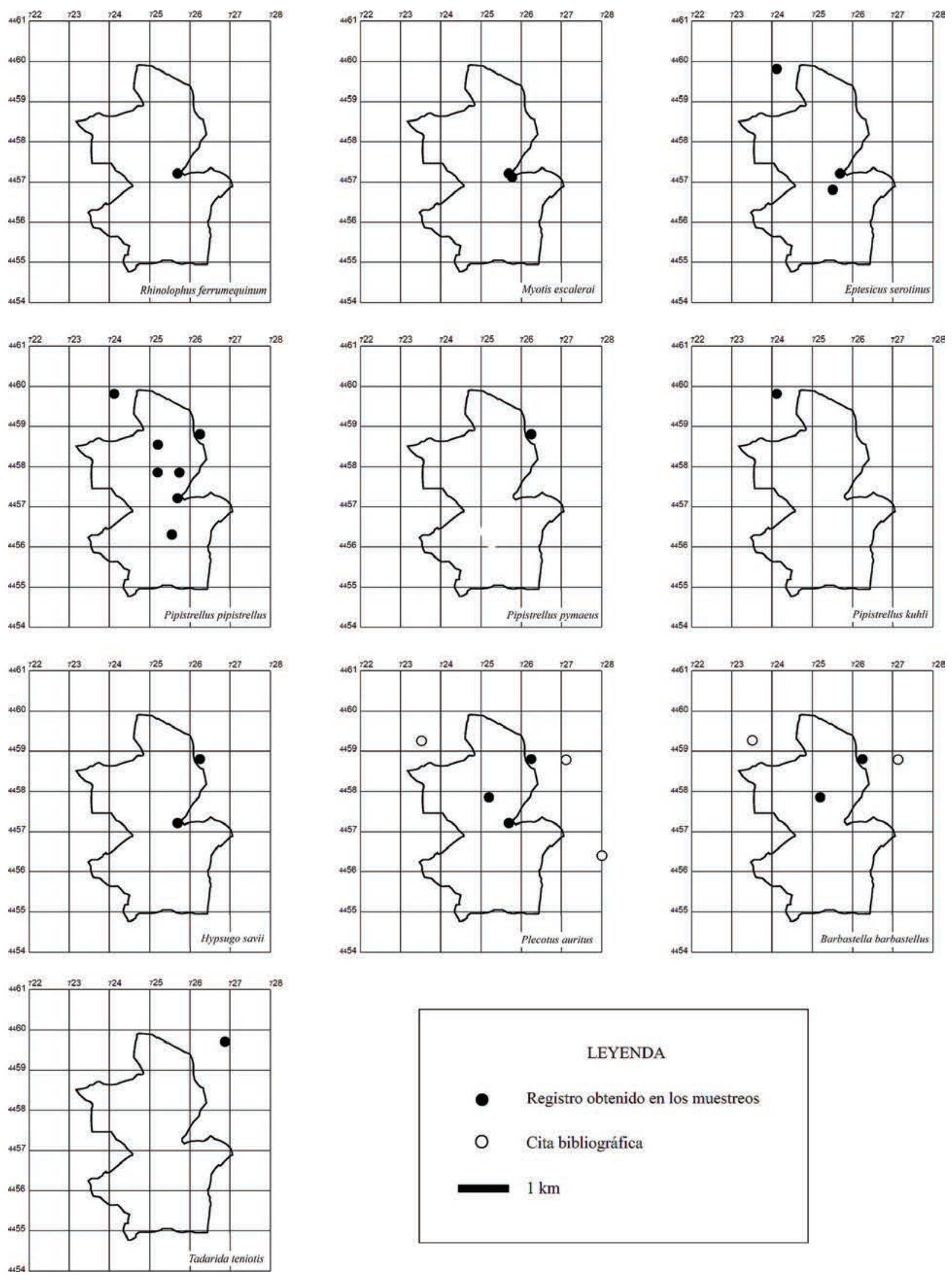

Fig. 2.- Mapas de localización de las especies registradas.

Fig. 2.- Location maps of the registered species. 


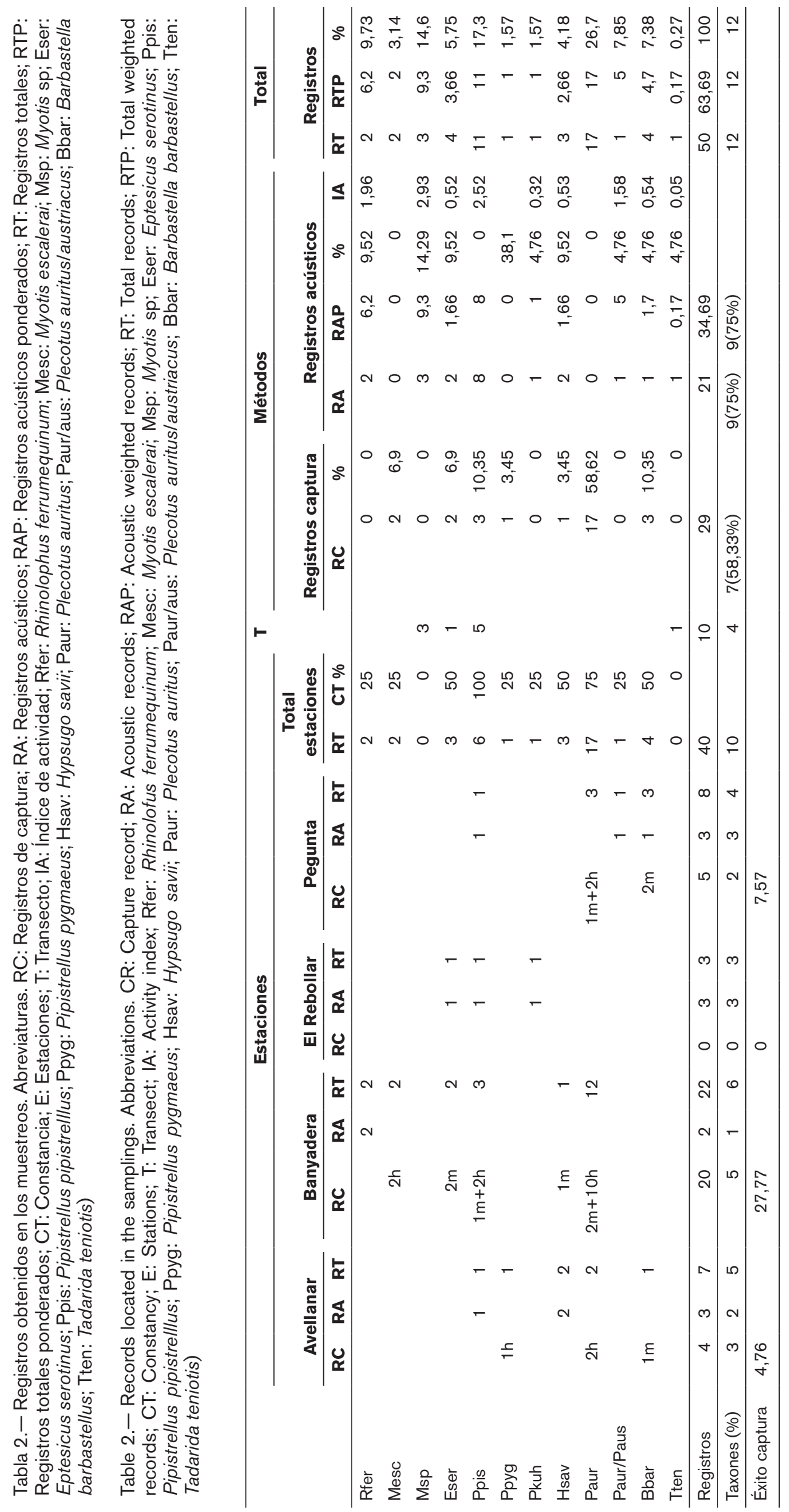




\section{ESPECIES FISURÍCOLAS}

En lo que respecta a las especies fisurícolas, en las estaciones, se han obtenido siete capturas, que representan el 24,15\% de las realizadas y siete registros acústicos. En el transecto, se obtuvieron otros siete registros acústicos que incrementaron hasta 14 el número de los contactos brutos.

Pipistrellus pipistrellus es la especie fisurícola localizada en mayor medida al haberse registrado en todas las estaciones de muestreo, así como en tres de las paradas del transecto acústico. La especie ha sido localizada mediante tres capturas, correspondientes al $10,35 \%$ de las realizadas y ocho registros acústicos, sobre los que se ha calculado un índice de actividad ponderado de 2,52 contactos/hora.

Eptesicus serotinus fue localizada a través de la captura de dos machos y dos registros acústicos.

Hypsugo savii fue registrado con la captura de un macho y la detección de dos registros acústicos.

Pipistrellus pygmaeus, Pipistrellus kuhli y Tadarida teniotis han sido localizadas con un solo registro cada una, una captura en el caso de la primera especie y un registro acústico en el caso de las dos restantes. Las tres especies fueron localizadas también a través de, respectivamente, tres, dos y dos registros acústicos en el casco urbano de Vistabella del Maestrat, última parada del transecto original (Albesa \& Ros, 2005).

\section{ESPECIES CAVERNICOLAS}

Por lo que concierne a las especies cavernícolas, fueron capturados dos ejemplares y detectados acústicamente otros dos.

Myotis escalerai, determinada inicialmente como Myotis nattereri (Kuhl, 1817) pero corregida a tenor de lo expuesto por Quetglas (2012), quien asigna a la primera especie todas las citas previas de la CV, fue observada a través de dos capturas. Tres registros acústicos de Myotis de pequeño tamaño se obtuvieron en el transecto. Estos registros, podrían corresponder a especies como M. emarginatus (É. Geoffroy, 1806), M. daubentonii (Kuhl, 1817), o M. mystacinus (Kuhl, 1817) entre otras o incrementar los registros de $M$. escalerai.

Rhinolophus ferrumequinum fue observada en una estación de muestreo mediante dos registros acústicos.

\section{Discusión}

Los muestreos efectuados en 2004 permitieron localizar las 10 especies Rhinolophus ferrumequinum, Myotis escalerai, Eptesicus serotinus, Pipistrellus pipistrellus, Pipistrellus pygmaeus, Pipistrellus kuhlii, Hypsugo savii, Plecotus auritus, Barbastella barbastellus y Tadarida teniotis más el par P. auritus/ austriacus y el grupo Myotis sp. Puesto que las únicas citas específicas publicadas correspondían a P. auritus y B. barbastellus (Almenar \& Alcocer, 2000), la publicación de los resultados obtenidos en los muestreos suponen incrementar en ocho el número de taxones de rango específico conocido hasta entonces. Por otra parte, la revisión de la bibliografía posterior sobre la zona (Monsalve et al., 2006; Alcocer, 2012); del Informe Técnico correspondiente a la revisión de las cajas nido (Servicio de Vida Silvestre, 2017) y de la memoria de gestión del Parque Natural correspondiente al año 2011 indican la presencia de tres especies más dentro de los límites del Parque Natural y de una más en una cuadrícula adyacente, Rhinolophus hipposideros (Bechstein, 1800), Myotis blythii (Tomes, 1856), Myotis emarginatus y Nyctalus leisleri (Kuhl, 1817). Las dos primeras son citadas en Monsalve et al. (2006), siendo citada también la primera en la memoria de gestión del Parque Natural junto con la tercera y citándose la última en Alcocer (2012) y en Servicio de Vida Silvestre (2017). Con todo, el número de especies localizadas en el Parque Natural o sus cuadrículas adyacentes se sitúa en al menos 14 .

Dos especies de las localizadas (Rhinolophus ferrumequinum y Barbastella barbastellus) y otras dos de las citadas (Myotis emarginatus y Rhinolophus hipposideros) están incluidas en el anexo II de la directiva hábitats (Directiva 92/42/CEE, de 21 de mayo) y una de las localizadas (Rhinolophus ferrumequinum) y otra de las citadas (Myotis emarginatus) en el Catálogo Español de Especies Amenazadas (Real Decreto 139/2011, de 4 de febrero).

Las especies forestales han sido las mayormente localizadas en los muestreos en consonancia con el carácter netamente forestal del entorno.

Plecotus auritus fue localizada por primera vez en el actual Parque Natural a partir de la localización de una colonia de cría en junio de 1998 (Albesa \& Ros, 2005; Albesa, 2012b) y citada en octubre del mismo año en cuadrículas adyacentes (Almenar \& Alcocer, 2000). La especie ha sido localizada con posterioridad en la zona (BDB, datos cedidos) y observada ocupando favorablemente cajas nido instaladas en la zona (Servicio de Vida Silvestre, 2017) en consonancia con el hecho de que se trata de la especie que utiliza en mayor medida este tipo de refugios (Benzal, 1990, 1991; Paz, 2007).

Barbastella barbastellus se encontraba citada en la CV por Boscá (1915), Bauer (1957) y Guerrero et al. (1988) y volvió a serlo por Almenar \& Alcocer (2000), quienes entre otras localidades del interior de la $\mathrm{CV}$ la citaron, a partir de la captura de seis machos y una hembra, en dos localidades del macizo de Penyagolosa cuya ubicación, aunque fuera de los límites del Parque Natural, corresponde a cuadrículas que son adyacentes.

Almenar \& Alcocer (2000) apuntaban la existencia de indicios de que $P$. auritus y $B$. barbastellus formasen poblaciones estables en el macizo de Penyagolosa. En el caso de $P$. auritus se indicaba que, al menos en el periodo otoñal, la especie era frecuente en Penyagolosa exhibiendo una desigual proporción sexual como consecuencia de la característica distribución espacial 
dispersa de los machos y agrupada de las hembras. Los datos que actualmente se poseen sobre $P$. auritus, se enmarcan en un rango temporal comprendido entre los meses de abril y de octubre. Las capturas concretas que se han realizado son 12 entre abril y mayo de 1999 (4 hembras), 2001 (5 hembras y 2 machos) y 2003 (1 macho), con una proporción de hembras del $75 \%$ (BDB, datos cedidos); 17 en agosto de 2005, con una proporción de hembras del 82,35\% (Albesa \& Ros, 2005) y tres machos en octubre de 1998 (Almenar \& Alcocer, 2000). Por otra parte, la especie ha sido localizada dentro de este rango temporal en dos refugios diurnos, uno en 1998 en una casa forestal próxima al Santuario (Albesa \& Ros, 2005) y otro en 2006 en el Forat de Falcó, cueva sita en una cuadrícula adyacente a las del Parque Natural que constituye el único refugio diurno conocido en una cavidad subterránea (Monsalve et al., 2006). La comparación con poblaciones de otros macizos montañosos como las sierras de Guadarrama (Benzal, 1991) y Alto Rey (Paz et al., 2000) permite observar unas presencias temporales similares y unas proporciones sexuales también desviadas hacia las hembras $(60,48 \%$ de hembras en el caso de Guadarrama y 74,32 \% en el caso de Alto Rey). Todo ello, unido al hecho de que se trata de una especie sedentaria cuyo mayor desplazamiento documentado es de $88 \mathrm{~km}$ (Gaisler et al., 2003 in Hutson et al., 2008) y la circunstancia de que las áreas de caza se encuentran habitualmente en las proximidades de los refugios (Entwistle et al., 1996; Rakhmatulina, 1998 in Rostovskaya et al., 2000), nos lleva a considerar a la población de Penyagolosa, al menos, como habitual en la zona. Con todo, sus efectivos deben ser muy reducidos sobre el total de las poblaciones nacionales y europeas, aunque su valor ecológico debe ser muy alto al tratarse de una población que, junto con las que posiblemente vivan en otras áreas montañosas del interior de Castellón y Valencia, habitaría en el límite meridional del área de distribución de la especie (Albesa, 2012b).

En el caso de B. barbastellus, se comentaba incluso la posibilidad de que la especie se reprodujese en la zona. Los datos obtenidos sobre $B$. barbastellus (tres machos capturados) son similares a los que, con seis machos y una hembra, suministran Almenar \& Alcocer (2000), amplían su distribución temporal, son coherentes con las observaciones realizadas por estos autores y, apoyados por el carácter sedentario de la especie (Paz et al., 1986; Hutterer et al., 2005), refuerzan la idea acerca de la situación de la especie en la zona. Ello, posee gran importancia en la medida que parece ser se trata de una especie relativamente escasa en la $\mathrm{CV}$, asociada a zonas de temperaturas bajas y grandes extensiones forestales del interior de la provincia de Castellón, de la cual sólo se han localizado dos poblaciones (Monsalve, 2012b).

Las especies fisurícolas constituyen el segundo grupo con más registros en los muestreos, destacando Pipistrellus pipistrellus y Eptesicus serotinus.
La primera por haber sido localizada en todas las estacionen y poseer el mayor índice de actividad entre los taxones de rango específico registrados acústicamente. Datos coherentes si se considera que, según Monsalve et al. (2003), se trata de una de las especies más abundantes en la CV, circunstancia que se da especialmente en las zonas de interior (Monsalve, 2012a). La segunda forma parte del complejo de especies $E$. serotinus/E. isabellinus, especies crípticas y alopátridas que podrían tener en la CV su límite de distribución al vivir la primera en su parte meridional y la segunda en la septentrional (Albesa, 2012a), lo que nos lleva a considerar provisionalmente como E. serotinus a los ejemplares de Penyagolosa. La especie destaca por la captura de sólo machos, dos en la campaña de 2004 que nos ocupa así como cuatro en 2001 (BDB, datos cedidos). Datos últimos que apoyan la, discutida por Albesa (2012a), segregación sexual que exhibe la especie en nuestras latitudes con la preferencia añadida de machos por las zonas más elevadas. Los registros de Penyagolosa, con una altitud máxima de 1.508 m.s.n.m., serían los más elevados de la CV.

Las especies cavernícolas son el grupo con menos registros de los tres considerados. Sin embargo, dicho valor puede estar infravalorado al no haberse efectuado muestreos en refugios. Destaca la especie Myotis escalerai con dos capturas en una de las estaciones y la presencia de una colonia de 300 individuos en una cavidad localizada en una cuadrícula adyacente al Parque Natural (Monsalve et al., 2006).

La estación de la Balsa de la Banyadera se ha revelado, con el 68,97 \% de las capturas y la localización del $50 \%$ del total de los taxones registrados en el Parque Natural, como la que mayores números ha proporcionado. Ello puede deberse a la simple presencia de una masa de agua que habitualmente puedan utilizar los murciélagos como bebedero, hecho observado en los muestreos y circunstancia que en los ecosistemas mediterráneos reviste especial importancia como apuntan Russo \& Jones (2003).

El análisis de los porcentajes en los que han sido capturadas las especies así como de los índices de actividad de las registradas acústicamente, sugieren la existencia de una comunidad en la que destacan las especies $P$. auritus y $P$. pipistrellus. Ello, es coherente con el carácter forestal del Parque Natural, por lo que se refiere a la primera especie y, sobre la segunda por su carácter oportunista y por ser una de las especies más abundantes en la CV. Con todo, los resultados y su análisis quedan condicionados por el reducido número de registros obtenidos. Prospecciones en refugios y ambientes no muestreados en profundidad en esta ocasión podrían detectar, cuanto menos, a las especies citadas por otros autores. Todo ello, sugiere la realización de nuevos muestreos que a la vez permitirían adquirir una valiosa información sobre la situación actual y posible evolución de las poblaciones con la consiguiente posibilidad de plantear medidas de conservación. 


\section{Agradecimientos}

Los autores quieren agradecer a la Conselleria de Territorio y Vivienda la concesión de los pertinentes permisos para efectuar los muestreos; a Susana Granell, Ángel García-Mochales, Paco Almodovar y Jose Carlos Fenollosa su valiosa participación en los muestreos; a Juan Casabó sus orientaciones sobre los sistemas de información geográfica y la lectura del manuscrito final y a Miguel Ángel Monsalve la revisión crítica del manuscrito y la cesión de los datos que sobre las especies forestales constan en el BDB así como de los registros existentes en algunos informes técnicos del Servicio de Vida Silvestre.

\section{Referencias}

Albesa, J., 2012a. Eptesicus serotinus. En: Jiménez, J., Monsalve, M.A. y Raga, J.A. (Eds.). Mamíferos de la Comunitat Valenciana. Colección Biodiversidad, 19. Conselleria d'Infraestructures, Territori i Medi Ambient, Generalitat Valenciana. Valencia: 231-235.

Albesa, J., 2012b. Plecotus auritus. En: Jiménez, J., Monsalve, M.A. y Raga, J.A. (Eds.). Mamiferos de la Comunitat Valenciana. Colección Biodiversidad, 19. Conselleria d'Infraestructures, Territori i Medi Ambient, Generalitat Valenciana. Valencia: 237-241.

Albesa, J. \& Ros, J., 2005. Inventario de los murciélagos de los macizos montañosos de Peñagolosa, Espadán y La Tinença de Benifassa. Conselleria de Territorio y Vivienda, Generalitat Valenciana. 24 pp. Inédito.

Alcocer, A., 2012. Nyctalus leisleri. En: Jiménez, J., Monsalve, M.A. y Raga, J.A. (Eds.). Mamíferos de la Comunitat Valenciana. Colección Biodiversidad, 19. Conselleria d'Infraestructures, Territori i Medi Ambient, Generalitat Valenciana. Valencia: 216-219.

Alcocer, A., Almenar, D. \& Monsalve, M. A., 2001. Estatus de los murciélagos forestales en dos zonas LIC de la Comunidad Valenciana. Conselleria de Medio Ambiente, Generalitat Valenciana. Inédito.

Alcocer, A., Castelló, A. J., Monsalve, M. A. \& Almenar, D., 2003. Nuevas citas de murciélagos forestales en la Comunidad Valenciana. Resúmenes II Jornadas sobre Estudio y Conservación de los Murciélagos, Valencia: 30.

Ahlén, I., 1990. Identification of Bats in Flight. Swedish society for conservation of nature \& the Swedish youth association for environmental studies and conservation. Stockholm. 50 pp.

Almenar, D. \& Alcocer, A., 1998. Los murciélagos forestales en la Comunidad Valenciana. Conselleria de Medio Ambiente. Generalitat Valenciana. Inédito.

Almenar, D. \& Alcocer, A., 2000. Nuevas citas de murciélagos forestales en la Comunidad Valenciana. Dugastella, 1: $35-38$

Ashrafi, S., Rutishauser, M., Ecker, K., Obrist, M. K., Arlettaz, R. \& Bontadina, F., 2013. Habitat selection of three cryptic Plecotus bat species in the European Alps reveals contrasting implications for conservation. Biodiversity and Conservation, 22: 2751-2766. https:// doi.org/10.1007/s10531-013-0551-z

Bauer, K., 1957. Zur kenntnis der Fledermausfauna der Spanien. Bonner Zoologische Beiträge, 7: 296-319.
Barataud, M., 1996. The World of Bats. Sittelle Press. Mens. 44 pp.

Barataud, M., 2002. Méthode d'identification acoustique des chiroptères de France. Mise à jour 2002. CD + livret. Edition Sittelle. Mens. 14 pp.

Barataud, M., 2012. Écologie acoustique des chiroptères d'Europe. Identification des espèces, études de leurs habitats et comportements de chasse. Muséum national d'Histoire naturelle (collection Inventaires et biodiversité). Biotope. Mèze. 344 pp.

Benzal, J., 1990. El uso de cajas anidaderas para aves por murciélagos forestales. Ecología, 4: 207-212

Benzal, J., 1991. Population dynamics of the brown longeared bat (Plecotus auritus) occupying bird boxes in a pine forest in central Spain. Netherlands Journal of Zoology, 41: 241-249.

Boscá, E., 1915. Comentarios sobre mamíferos de la región valenciana comprendidos en la "Fauna Ibérica" de D. Angel Cabrera. Memorias de la Real Sociedad Española de Historia Natural, 10: 125-146.

Dietz, C. \& von Helversen, O. 2004. Illustrated identification key to the bats of Europe. Electronic Publication, version 1.0. Disponible en: https://www.researchgate. net/publication/228985859 Illustrated identification key_to_the_bats_of_Europe [consultada 20.4.2005].

Entwistle, A. C., Racey, P. A. \& Speakman, J. R., 1996. Habitat exploitation by a gleaning bat, Plecotus auritus. Philosophical Transactions of the Royal Society of London, Series B, 351: 921-931. https://doi.org/ 10.1098/rstb.1996.0085

Fabregat Llueca, C., Aparicio, J. M. \& Andrés, J. V., 2005. Aportaciones a la flora del macizo de Penyagolosa (Castellón). Toll Negre, 6: 42-44.

Flaquer, C., Torre, I. \& Arrizabalaga. A., 2007. Comparison of sampling methods for inventory of bat communities. Journal of Mammalogy, 88(2): 526-533. https://doi. org/10.1644/06-MAMM-A-135R1.1

Font Bustos, J.M., 1978. Capturas más interesantes efectuadas en la provincia de Castellón. SHILAP Revista de lepidopterología, 6(24): 324.

Gaisler, J., Hanák, V., Hanzal, V. \& Jarský, V., 2003. [Results of bat banding in the Czech and Slovak Republics, 1948-2000.] Vespertilio, 7: 3-61 (en Checo).

Guerrero, S. L., Guillén-Servent, A., Ibáñez-Ulargui, C., Pérez-Jordá, J. L. \& Ruiz-Benavides, C., 1988, Contribución al conocimiento de la fauna de quirópteros de la Comunidad Valenciana. Conselleria de Agricultura y Pesca de la Generalitat Valenciana y Estación Biológica de Doñana (CSIC). Informe inédito.

Hutson, A. M., Spitzenberger, F., Aulagnier, S., Coroiu, I., Karataş, A., Juste, J., Paunovic, M., Palmeirim, J. \& Benda, P., 2008. Plecotus auritus. In: IUCN 2010. IUCN Red List of Threatened Species. Version 2010.4. https://doi.org/10.2305/IUCN.UK.2008.RLTS. T17596A7154745.en

Hutterer, R., Ivanova, T., Meyer-Cords, C. \& Rodrigues, L., 2005. Bat migrations in Europe: a review of banding data and literature. Naturschutz und Biologische Vielfalt Vol. 28. Federal Agency for Nature Conservation. Bonn. $176 \mathrm{pp}$. 
Jiménez, J., Monsalve, M. A. \& Raga, J. A. (Eds.), 2012. Mamíferos de la Comunitat Valenciana. Colección Biodiversidad, 19. Conselleria d'Infraestructures, Territori i Medi Ambient, Generalitat Valenciana. Valencia. 299 pp.

Monsalve, M.A., 2012a. Pipistrellus pipistrellus. En: Jiménez, J., Monsalve, M.A. y Raga, J.A. (Eds.). Mamíferos de la Comunitat Valenciana. Colección Biodiversidad, 19. Conselleria d'Infraestructures, Territori i Medi Ambient, Generalitat Valenciana. Valencia: 219-221.

Monsalve, M.A., 2012b. Barbastella barbastellus. En: Jiménez, J., Monsalve, M.A. y Raga, J.A. (Eds.). Mamíferos de la Comunitat Valenciana. Colección Biodiversidad, 19. Conselleria d'Infraestructures, Territori i Medi Ambient, Generalitat Valenciana. Valencia: 235-237.

Monsalve, M. A., Almenar, D., Alcocer, A. \& Castelló, A., 2003. Los murciélagos de la Comunidad Valenciana. Sedeck, 4: 22-33.

Monsalve, M. A., Castelló, A., Alcocer, A. \& Almenar, D., 2006. Forat del Falcó. Ficha descriptiva. Conselleria de Territorio y Vivienda. Disponible en: http://www. agroambient.gva.es/estatico/areas/espacios/cuevas/ forat_del_falco.pdf [consultado 28.8.2017].

Palomo, L. J., Gisbert, J. \& Blanco, J. C. (Eds.), 2007. Atlas y libro rojo de los mamíferos terrestres de España. Dirección General para la Biodiversidad-SECEMSECEMU. Madrid. 586 pp.

Paz, O. de, 2007. Plecotus auritus Linnaeus, 1758. In: Palomo, L. J., Gisbert, J. \& Blanco, J. C. (Eds.). Atlas y Libro Rojo de los Mamíferos Terrestres de España. Dirección General para la Biodiversidad-SECEMSECEMU. Madrid: 246-249.

Paz, O. de, Fernández, R. \& Benzal, J., 1986. El anillamiento de Quirópteros en el centro de la Península Ibérica durante el período 1977-1986. Boletín de la Estación Central de Ecología, 30: 113-138.

Paz O. de, Lucas J. \& Arias J., 2000. Cajas refugio para quirópteros y estudio de la población del murciélago orejudo dorado (Plecotus auritus Linneo, 1758) en un área forestal de la provincia de Guadalajara. Ecología, 14: 259-268.

Pérez y de Lanuza, G., Luna, S. \& Lozano, J., 2012. Actualización de la distribución de Podarcis muralis en el Penyagolosa (País Valenciano). Boletín de la Asociación Herpetológica Española, 23(1): 73-79.

Quetglas, J., 2012. Myotis escalerai. En: Jiménez, J., Monsalve, M.A. y Raga, J.A. (Eds.). Mamíferos de la Comunitat Valenciana. Colección Biodiversidad, 19. Conselleria d'Infraestructures, Territori i Medi Ambient, Generalitat Valenciana. Valencia: 203-206.

Rakhmatulina, I. K., 1998. [Daily activity patterns of bats in Eastern Transcaucasia.] Plecotus et al.,1: 35-47 (en Ruso).

Rostovskaya, M. S., Zhukova, D. V., Illarionova, A. E., Ustyugova, S. V., Borissenko, A. V. \& Sviridov, A. V., 2000. Insect prey of the Long-eared bat Plecotus auritus (L.) (Chiroptera: Vespertilionidae) in Central Russia. Russian Entomological Journal, 9(2): 185-189.

Russo, D. \& Jones, G., 2002. Identification of twentytwo bat species (Mammalia: Chiroptera) from Italy by analysis of time-expanded recordings of echolocation calls. Journal of Zoology, London, 258: 91-103.

Russo, D. \& Jones, G., 2003. Use of foraging habitats by bats in a Mediterranean área determined by acoustic surveys: conservation implications. Ecography, 26: 197-209.

Schober, W. \& E. Grimmberger, E., 1996. Los murciélagos de España y de Europa. Ediciones Omega. Barcelona. $237 \mathrm{pp}$.

Servicio de Vida Silvestre, 2017. Revisión de cajas-refugio para murciélagos en zonas Forestales de la comunidad valenciana. Año 2017. Conselleria de Agricultura, Medi Ambient, Canvi Climàtic i Desenvolupament Rural, Generalitat Valenciana. 12 pp. Inédito.

Vigo, J., 1968. La vegetació del massis de Penyagolosa. Institut d'Estudis Catalans, Arxius de la Secció de Ciencies, 37. Barcelona. $246 \mathrm{pp}$. 